\title{
Generating matrices of the $k$-Jacobsthal Numbers
}

\author{
G.Srividhya ${ }^{1}$ and T.Ragunathan ${ }^{2}$ \\ 1. Assistant professor, Department of Mathematics, Government Arts College, Trichy-22, Tamilnadu, India. e-mail \\ :vkm292011@hotmail.com \\ 2. Assistant professor, Department of Mathematics, PGP College of Arts and Science, Namakkal. Tamilnadu, India. \\ e-mail : math_ragu@rediffmail.com
}

\begin{abstract}
In this paper we define some tridiagonal matrices depending of a parameter from which we will find the $k$-jacobsthal numbers. And from the cofactor matrix of one of these matrices we will prove some formulas for the $k$ jacobsthal numbers differently to the traditional form. Finally we will study the eigenvalues of these tridiagonal matrices.
\end{abstract}

\section{INTRODUCTION}

The generalization of the Fibonacci has been treated by some authors eg. Hoggat V.E and Horadom A.F. k- Fibonacci generalizations has been found by Falcon .S and plaza A to study the method of triangulation.

In this paper we have to give generating matrices of the $k$-jacobsthal numbers Besides the usual jacobsthal numbers many kinds of generalization of these have been presented and well known jacobsthal sequence is defined as $j_{0}=0, j_{1}=$ $1, j_{n}=j_{n-1}+2 j_{n-2}$, for $n \geq 2$ where $j_{n}$ denotes the $\mathrm{n}$-jacobsthal numbers for any positive real number $\mathrm{k}$, the k-jacobsthal sequence say $\left\{j_{k, n}\right\}_{n=0}^{\infty}$ is defined recurrently by

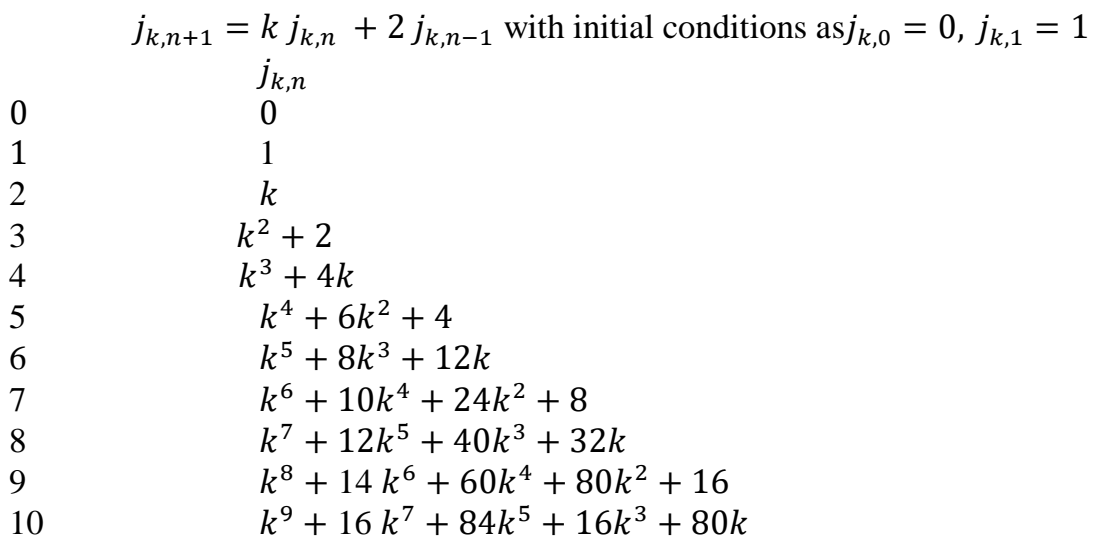

Particular cases of the previous definitions are if $\mathrm{k}=1$, the classical jacobsthal sequence obtained $j_{0}=0, j_{1}=$ $1, j_{n+1}=j_{n}+2 j_{n-1}$ for $n \geq 1,\left\{j_{k, n}\right\}_{n=0}^{\infty}=\{0,1,1,3,5,11 \ldots\}$

\section{Tridiagonal matrices and k-jacobsthal numbers}

In this section we extend the matrices defined and applied them to the $\mathrm{k}$-jacobsthal numbers in order to prove some formulas differently to the traditional form.

\subsection{The determinant of a special kind of the tridiagonal matrices}

Let us consider the $\mathrm{n}$ by $\mathrm{n}$ tridiagonal matrices 


$$
M_{n}=\left(\begin{array}{ccccccc}
a & b & & & & & \\
c & d & e & & & & \\
& c & d & e & & & \\
& & \ddots & \ddots & \ddots & & \\
& & & & c & d & e \\
& & & & & c & d
\end{array}\right)
$$

Solving the sequence of determinants, we find

$$
\begin{aligned}
& \left|M_{1}\right|=a \\
& \left|M_{2}\right|=d .\left|M_{1}\right|-b c \\
& \left|M_{3}\right|=d .\left|M_{2}\right|-c e .\left|M_{1}\right| \\
& \left|M_{4}\right|=d .\left|M_{3}\right|-c e .\left|M_{2}\right|
\end{aligned}
$$

In general

$$
\cdots
$$

$$
\left|M_{n+1}\right|=d \cdot\left|M_{n}\right|-c e \cdot\left|M_{n-1}\right|
$$

2.2 Some tridiagonal matrices and $k$-jacobsthal numbers

$$
\text { If } a=d=k, b=e=2 \text { and } c=-1 \text {, the matrices Mn transformed in the tridiagonal matrices }
$$

$$
H_{n}(k)=\left(\begin{array}{ccccccc}
k & 2 & & & & & \\
-1 & k & 2 & & & & \\
& -1 & k & 2 & & & \\
& & \ddots & \ddots & \ddots & & \\
& & & & -1 & k & 2 \\
& & & & & -1 & k
\end{array}\right)
$$

In this case and taking

$$
\begin{aligned}
& \left|H_{1}(k)\right|=k=j_{k, 2} \\
& \left|H_{2}(k)\right|=k \cdot k-2(-1)=k^{2}+2=j_{k, 3} \\
& \left|H_{3}(k)\right|=k \cdot\left(k^{2}+2\right)-2(-1) \cdot k=k^{3}+4 k=j_{k, 4}
\end{aligned}
$$

And formula (2.1) is $\left|H_{1}(k)\right|=j_{k, n+1}$ for $n \geq 1$

The k-jacobsthal numbers can also be obtained from the symmetric tridiagonal matrices

$$
H_{n}^{\prime}(k)=\left(\begin{array}{ccccccc}
k & i & & & & & \\
i & k & i & & & & \\
& i & k & i & & & \\
& & \ddots & \ddots & \ddots & & \\
& & & & i & k & i \\
& & & & & i & k
\end{array}\right)
$$


Where $i$ is the imaginary unit,i.e $i^{2}=-1$

* if $a=k^{2}+2 d=k^{2}+4, \quad b=e=c=2$, the tridiagonal matrices are $O_{n}(k)=\left(\begin{array}{ccccccc}k^{2}+2 & 2 & & & & & \\ 2 & k^{2}+4 & 2 & & & & \\ & 2 & k^{2}+4 & 2 & & & \\ & & \ddots & \ddots & \ddots & & \\ & & & & 2 & k^{2}+4 & 2 \\ & & & & & 2 & k^{2}+4\end{array}\right)$

In this case, it is

$\left|O_{n}(k)\right|=j_{k, 2 n+1}$ for $n \geq 1$ So, with $\left|O_{0}(k)\right|=j_{k, 1}=1$, the sequence of these determinants is the sequence of odd $k$-jacobsthal numbers $\left\{1, k^{2}+2, k^{4}+6 k^{2}+4, k^{6}+10 k^{4}+24 k^{2}+4 \ldots.\right\}$

*Finally If $a=k d=k^{2}+4, \quad b=0, e=c=2$ for $n \geq 1$

$\left|E_{n}(k)\right|=\left(\begin{array}{ccccccc}k & 0 & & & & & \\ 2 & k^{2}+4 & 2 & & & & \\ & 2 & k^{2}+4 & 2 & & & \\ & & \ddots & \ddots & \ddots & & \\ & & & & 2 & k^{2}+4 & 2 \\ & & & & & 2 & k^{2}+4\end{array}\right)$

because $\left|E_{n}(k)\right|=j_{k, 2 n}$ for $n \geq 1$ So, with $\left|E_{0}(k)\right|=j_{k, 0}=0$

Cofactor matrices of the generating matrices of the k-jacobsthal numbers

The following definitions are well Known:

If $\mathrm{A}$ is a square matrix, then the minor of its entry $a_{i j}$, also known as the $(i, j)$ minor of $\mathrm{A}$, is denoted by $M_{i j}$ and is defined to be the determinant of the submatrix obtained by removing from A its $i-$ th row and $j-$ th column.

If follows $C_{i j}=(-1)^{i+j} M_{i j}$ and $C_{i j}$ called the cofactor of $a_{i j}$, also refered to as the $(i, j)$ cofactor of A.
Define the cofactor matrix of A,as the $n \times n$ matrix $C$ whose $(i, j)$ entry is the $(i, j)$ cofactor of A.

Finally, the inverse matrix of $\mathrm{A}$ is $A^{-1}=\frac{1}{|A|} C^{T}$, where $|A|$ is the determinant of the matrix $\mathrm{A}$ (assuming non zero) and $C^{T}$ is the transpose of the cofactor matrix $\mathrm{C}$ or adjugate matrix of $\mathrm{A}$.

On the other hand, let us consider the $n X n$ nonsingular tridiagonal matrix 


$$
\mathrm{T}=\left(\begin{array}{ccccccc}
a_{1} & b_{1} & & & & & \\
c_{1} & a_{2} & b_{2} & & & & \\
& c_{2} & a_{3} & b_{3} & & & \\
& & \ddots & \ddots & \ddots & & \\
& & & & c_{n-2} & a_{n-1} & b_{n-1} \\
& & & & & c_{n-1} & a_{n}
\end{array}\right)
$$

In an elegant and coincise formula for the inverse of the tridiagonal matrix $T^{l}=\left(t_{i, j}\right)$ :

where

$$
\mathrm{t}_{\mathrm{ij}}= \begin{cases}(-1)^{i+j} \frac{1}{\theta_{n}} b_{i} \ldots \ldots b_{j-1} \theta_{i-1} \varphi_{j+1} & \text { if } i \leq j \\ (-1)^{i+j} \frac{1}{\theta_{n}} c_{j} \ldots \ldots . c_{i-1} \theta_{j-1} \varphi_{i+1} & \text { if } i>j\end{cases}
$$

$\theta_{\mathrm{i}}$ verify the recurrence relation $\theta_{i}=a_{i}^{\mathrm{T}} \boldsymbol{\theta}_{i-1}^{\mathrm{pe}} \mathrm{e} b_{i-1}^{\mathrm{ati}} \theta_{i-2}$ herfor $i=2, \ldots \ldots n$ with the initial conditions $\theta_{0}=1$ and $\theta_{1}=a_{1}$.

Formula (2.1) is one special case of this one.

$\varphi_{i}$ verify the recurrence relation $\varphi_{i}=a_{i} \theta_{i+1}-b_{i} c_{i} \varphi_{i+2}$ for $i=n-1, \ldots \ldots 1$ with the initial conditions $\varphi_{n+1}=1$ and $\varphi_{n}=a_{n}$.observe that $\varphi_{n}=\operatorname{det}(T)$.

\subsection{Cofactor matrix of $\boldsymbol{H}_{\boldsymbol{n}}(\boldsymbol{k})$}

For the matrix $H_{n}(k)$, it is $a_{i}=k, b_{i}=2, c_{i}=-1 \theta_{i}=j_{k, i+1}$ and $\theta_{i}=j_{k, n-j+2}$

Consequently

$$
\left(\left(H_{n}(k)\right)^{-1}\right)_{i, j}=\left\{\begin{array}{c}
(-1)^{i+j} \frac{1}{j_{k, n+1}} j_{k, i} \cdot j_{k, n-j+1} \quad \text { if } i \leq j \\
\frac{1}{j_{k, n+1}} j_{k, j} \cdot j_{k, n-i+1} \quad \text { if } i>j
\end{array}\right.
$$

We will work with the cofactor matrix whose entries are

$$
c_{i, j}\left(H_{n}(k)\right)=\left\{\begin{array}{c}
(-1)^{i+j} j_{k, j} \cdot j_{k, n-i+1} \quad \text { if } i \geq j \\
j_{k, i} \cdot j_{k, n-j+1} \text { if } i<j
\end{array}\right.
$$

So $c_{j, i}\left(H_{n}(k)\right)=(-1)^{i+j} c_{i, j}\left(H_{n}(k)\right)$ if $i>j$

In this form, the cofactor matrix of $H_{n}(k)$ for $n \geq 2$ is

$$
C_{n-1}(k)=\left(\begin{array}{ccccccc}
j_{k, n} & 2 j_{k, n-1} & j_{k, n-2} & j_{k, n-3} & \ldots & j_{k, 2} & j_{k, 1} \\
-j_{k, n-1} & j_{k, 2} j_{k, n-1} & 2 j_{k, 2} j_{k, n-2} & j_{k, 2} j_{k, n-3} & \ldots & j_{k, 2} j_{k, 2} & j_{k, 2} \\
j_{k, n-2} & -j_{k, 2} j_{k, n-2} & j_{k, 3} j_{k, n-2} & 2 j_{k, 3} j_{k, n-3} & \ldots & j_{k, 3} j_{k, 2} & j_{k, 3} \\
-j_{k, n-3} & j_{k, 2} j_{k, n-3} & -j_{k, 3} j_{k, n-3} & j_{k, 4} j_{k, n-3} & \ldots & j_{k, 4} j_{k, 2} & j_{k, 4} \\
\ldots & \ldots & \ldots & \ldots & \ldots & \ldots & \ldots \\
j_{k, 2} & -j_{k, 2} j_{k, 2} & j_{k, 3} j_{k, 2} & -j_{k, 4} j_{k, 2} & \ldots & j_{k, n-1} j_{k, 2} & 2 j_{k, n-1} \\
-j_{k, 1} & j_{k, 2} & -j_{k, 3} & j_{k, 4} & \ldots & -j_{k, n-1} & j_{k, n}
\end{array}\right)
$$

On the other hand taking into account of the inverse matrix $A^{-1}=\frac{1}{|A|} \operatorname{Adj}(A)$,

It is easy to prove $|\operatorname{Adj}(A)|=A^{n-1}$ 


\section{Available online at www.ijrat.org}

$$
\begin{aligned}
& \text { So }\left|C_{n-1}\right|=j_{k, n+1}^{n-1} \\
& \text { In this form, for } n=2,3,4 \ldots \text { It is } \\
& C_{1}(k)=\left|\begin{array}{cc}
j_{k, 2} & 2 j_{k, 1} \\
-j_{k, 1} & j_{k, 2}
\end{array}\right|=j_{k, 3} \rightarrow j_{k, 2}^{2}+2 j_{k, 1}^{2}=j_{k, 3} \\
& C_{2}(k)=\left|\begin{array}{ccc}
j_{k, 3} & 2 j_{k, 2} & j_{k, 1} \\
-j_{k, 2} & j_{k, 2} j_{k, 2} & 2 j_{k, 2} \\
j_{k, 1} & -j_{k, 2} & j_{k, 3}
\end{array}\right|=j_{k, 4}^{2} \\
& \rightarrow j_{k, 2}^{2}\left(j_{k, 3}^{2}+4 j_{k, 3}^{2}+4\right)=j_{k, 4}^{2} \\
& \rightarrow\left(\frac{j_{k, 3-2 j_{k, 1}}}{k}\right)^{2}\left(j_{k, 3}+2 j_{k, 1}\right)^{2}=j_{k, 4}^{2} \\
& \rightarrow j_{k, 3}^{2}-2 j_{k, 1}^{2}=k j_{k, 4} \\
& C_{3}(k)=\left|\begin{array}{cccc}
j_{k, 4} & 2 j_{k, 3} & j_{k, 2} & j_{k, 1} \\
-j_{k, 3} & j_{k, 3} j_{k, 2} & 2 j_{k, 2} j_{k, 2} & j_{k, 2} \\
j_{k, 2} & -j_{k, 2} j_{k, 2} & j_{k, 3} j_{k, 2} & 2 j_{k, 3} \\
-j_{k, 1} & j_{k, 2} & -j_{k, 3} & j_{k, 4}
\end{array}\right|=j_{k, 5}^{3} \rightarrow j_{k, 5}^{2}\left(j_{k, 3}^{2}+2 j_{k, 2}^{2}\right)=j_{k, 5}^{3} \\
& \rightarrow\left(j_{k, 3}^{2}+2 j_{k, 2}^{2}\right)=j_{k, 5} \\
& C_{4}(k)=j_{k, 6}^{4} \rightarrow j_{k, 3}^{2}\left(j_{k, 4}^{2}+4 j_{k, 2}^{2}\right) j_{k, 6}^{2}=j_{k, 6}^{4} \\
& \rightarrow\left(\frac{j_{k, 4-2 j_{k, 2}}}{k}\right)^{2}\left(j_{k, 4-2 j_{k, 2}}\right)^{2}=j_{k, 6}^{2} \\
& \rightarrow j_{k, 4}^{2}-2 j_{k, 2}^{2}=k j_{k, 6}
\end{aligned}
$$

Generating these results and taking into account $j_{k, n}=\frac{j_{k, n+1}-2 j_{k, n-1}}{k}$, we find the following two formulas for kjacobsthal numbers according to that $\mathrm{n}$ is odd or even $j_{k, n+1}^{2}+2 j_{k, n}^{2}=j_{k, 2 n+1}$ and $j_{k, n+1}^{2}-2 j_{k, n-1}^{2}=k j_{k, 2 n}$.

\subsection{Cofactor matrix of $\boldsymbol{O}_{\boldsymbol{n}}(\mathrm{k})$}

To apply (3.2) to the matrices $O_{n}(k)$, we must take into account that

$$
\begin{aligned}
& a_{1}=k^{2}+2 \\
& a_{i}=k^{2}+4 \quad i \geq 2
\end{aligned}
$$$$
b_{i}=c_{i}=2 \quad i \geq 1
$$$$
\theta_{i}=j_{k, 2 i-1}, i \geq 1
$$

$\varphi_{j}=\frac{1}{k} j_{k, 2(n-i+2),} j \geq 1$ and consequently the cofactor of the $(i, j)$ entry of these matrices is

$c_{i, j}\left(O_{n}(k)\right)=(-1)^{i+j} \frac{1}{k} j_{k, 2 j-1} j_{k, 2(n-i+1),} \quad i \geq j$

$c_{j, i}\left(O_{n}(k)\right)=c_{i, j}\left(O_{n}(k)\right)$ for $j \geq i$

\subsection{Cofactor matrix of $E_{n}(k)$}

For the matrices $E_{n}(k)$, we must take into account that

$$
\begin{gathered}
a_{1}=k=j_{k, 2} \\
a_{i}=k^{2}+4 \quad i \geq 2 \\
b_{1}=0 \\
b_{i+1}=c_{i}=2 \quad i \geq 1
\end{gathered}
$$




\section{Available online at www.ijrat.org}

$$
\begin{aligned}
\theta_{i} & =j_{k, 2(i+1)}, i \geq 1 \\
\varphi_{j} & =\frac{1}{k} j_{k, 2(n-i+2),} \quad j \geq 1 \text { and consequently the cofactor of the }(i, j) \text { entry of these matrices is } \\
c_{1, j}\left(E_{n}(k)\right) & =(-1)^{i+j} \frac{1}{k} j_{k, 2(n-i+1),} \\
c_{i, j}\left(E_{n}(k)\right) & =(-1)^{i+j} \frac{1}{k} j_{k, 2 j} j_{k, 2(n-i+1),} \quad i \geq j \\
c_{j, i}\left(E_{n}(k)\right) & =c_{i, j}\left(E_{n}(k)\right) \text { for } j>i>1
\end{aligned}
$$

\section{Eigen Values}

This section is dedicated to the study of the eigen values of the matrices $H_{n}(k), O_{n}(k)$ and $E_{n}(k)$.

\subsection{Eigen Values of the matrices $H_{n}(k)$}

The matrix has entries in the diagonals $a_{1}, a_{2}, \ldots . a_{n,}, b_{1}, b_{2}, \ldots . b_{n-1}, c_{1}, c_{2}, \ldots . c_{n-1}$

It is well known the eigen values of the matrix are

$\lambda_{r}=a+2 \sqrt{b c} \cos \left(\frac{r \pi}{n+1}\right)$ for $\mathrm{r}=1,2,3, \ldots, \mathrm{n}$.

Consequently, the eigen values of the matrix $H_{n}(k)$ where $\mathrm{a}=\mathrm{k}, \mathrm{b}=2, \mathrm{c}=-1$ are $\lambda_{r}=k+2 i \sqrt{2} \cos \left(\frac{r \pi}{n+1}\right)$.

If $\mathrm{n}$ is odd, then the matrix $H_{n}(k)$ has one unique real eigenvalue corresponding to $\mathrm{r}=\frac{n+1}{2}$.

If $\mathrm{n}$ is even, no one eigen value is real.

So, the sequence of the tridiagonal $H_{n}(k)$ for $\mathrm{n}=1,2, \ldots$ is

$$
\begin{gathered}
\sum_{1}=\{k\} \\
\sum_{2}=\{k-i \sqrt{2}\} \\
\sum_{3}=\{k, k-2 i\} \\
\ldots \ldots \ldots \ldots
\end{gathered}
$$

It is verified that $\sum_{j=1}^{n} \lambda_{j}=n k$ and $\prod_{j=1}^{n} \lambda_{j}=j_{k, n+1}$ where $j_{k, n+1}=\prod_{j=1}^{n}\left(k+2 i \sqrt{2} \cos \frac{\pi j}{n+1}\right)$.

\section{Eigen values of the Matrices $O_{n}(k)$}

Matrices $O_{n}(k)$ are symmetric, so all its eigenvalues are real.

\section{Theorem:}

If $\lambda_{i}$ is an eigenvalue of the matrix $O_{n}(k)$ for a fixed value $\mathrm{k}$, then $\lambda_{i}+2 k+1$ is eigenvalue of the matrix $O_{n}(k+$ 1).

\section{Proof:}

If $\lambda_{i}$ is an eigenvalue of the matrix $O_{n}(k)$, then it is

$$
\begin{aligned}
& =\left[\begin{array}{ccc}
(k+1)^{2}-\left(\lambda_{i}+2 k+1\right)-\lambda_{i} I_{n} \mid=\left[\begin{array}{ccc}
k^{2}+2-\lambda_{i} & 2 & \\
2 & k^{2}+4-\lambda_{i} & 2 \\
2 & k^{2}+4-\lambda_{i}
\end{array}\right] \\
2(k+1)^{2}+2-\left(\lambda_{i}+2 k+1\right) \\
2 & (k+1)^{2}+2-\lambda_{i}+2 k+1
\end{array}\right] \\
& =\left|O_{n}(k+1)-\left(\lambda_{i}+2 k+1\right) I_{n}\right|
\end{aligned}
$$




\section{International Journal of Research in Advent Technology, Vol.7, No.1, January 2019 E-ISSN: 2321-9637 \\ Available online at www.ijrat.org}

Consequently, only it is necessary to find the eigenvalues of the matrix $O_{n}(1)$ for $\mathrm{n}=2,3, \ldots$ and then,

if $\lambda_{j}$ is an eigenvalue of $O_{n}(1)$, then $\lambda_{j}^{\prime}=\lambda_{j}+k^{2}-1$ is an eigenvalue of the matrix $O_{n}(k)$.

\section{Eigen values of the matrices $E_{n}(k)$}

Finally, we say a matrix is positive if all entries are real and non negative. If a matrix is tridiagonal and positive, then all the eigen values are real. So taking into account matrix $E_{n}(k)$ is tridiagonal and positive, all its eigenvalues are real.

Following the same process that for the matrices $O_{n}(k)$, we can prove that the first eigen value is $\mathrm{k}$ and othes verify $\lambda_{i}=\lambda_{i}(1)+k^{2}-1$.

Moreover $\sum_{j=1}^{n} \lambda_{j}(k)=(n-1)\left(k^{2}+4\right)+k$ and $\prod_{j=1}^{n} \lambda_{j}(k)=j_{k, 2 n}$

\section{REFERENCES}

[1] Falcon S. and Plaza A., On the Fibonacci knumbers, Chaos, Solit. \& Fract.32 (5), pp. 1615-1624, (2007).

[2] Falcon.s, On the generating matrices of the kFibonacci numbers, Proyecciones Journal of Mathematics,Vol. 32, No 4, pp. 347-357, December 2013.

[3] Falcon S. and Plaza A., The k-Fibonacci sequence and the Pascal 2-triangle,Chaos, Solit. \& Fract. 33 (1), pp. 38-49, (2007).

[4] Feng A., Fibonacci identities via determinant of tridiagonal matrix, Applied Mathematics and Computation, 217, pp. 5978-5981, (2011).

[5] Horadam A. F. A generalized Fibonacci sequence, Mathematics Magazine,68, pp. 455459, (1961).

[6] Usmani R., Inversion of a tridiagonal Jacobi matrix, Linear Algebra Appl. 212/213, pp. 413414, (1994).

[7] Uygun.S, and Eldogan.H, Some properties of the $k$-Jacobsthal Lucas sequence Gen.Math.Notes, vol.36,No.1, pp 34-37.

[8] Srividhya G and Ragunathan T, Sequences of Diophantine Triples for $K$-Jacobsthal and $K$ Jacobsthal Lucas, International Journal of Pure and Applied Mathematics Volume 117 No. 12 2017, 431-439. 